Research Article

\title{
Sports Action Recognition Based on Image Processing Technology and Analysis of the Development of Sports Industry Pattern
}

\author{
Zhaoyin Jiang, ${ }^{1,2}$ Fuyou Zhang, ${ }^{3}$ and Laishuang Sun ${ }^{4}{ }^{4}$ \\ ${ }^{1}$ Physical Education Department of Jeonju University, Jeonju 55069, Republic of Korea \\ ${ }^{2}$ College of Sports and Leisure, Chengdu Sport University, Chengdu 610041, China \\ ${ }^{3}$ College of Sports and Leisure, Sichuan Tourism University, Sichuan 610100, China \\ ${ }^{4}$ Social Sports College of Shenyang Sport University, Shenyang 110102, China \\ Correspondence should be addressed to Laishuang Sun; 100989@cdsu.edu.cn
}

Received 10 November 2021; Revised 8 December 2021; Accepted 13 December 2021; Published 24 December 2021

Academic Editor: Fazli Wahid

Copyright $\odot 2021$ Zhaoyin Jiang et al. This is an open access article distributed under the Creative Commons Attribution License, which permits unrestricted use, distribution, and reproduction in any medium, provided the original work is properly cited.

\begin{abstract}
The current era is an information age, and society is turning to the information age. The image processing technology is also widely used in various fields, and the technology of sports action recognition based on image processing technology can also be said to be appropriate. This article uses a spatial visual feature analysis algorithm to implement it. To implement this algorithm, a series of work such as image collection, feature extraction, and action recognition must be completed first and then implemented through texture functions and other related functions. This algorithm can be used to complete the image-based sports action recognition technology at the minimum time cost. This algorithm can help sportsmen better complete training and standardize movements to a certain extent. As for the development of China's current sports industry structure, it is also steadily improving. The people's love for sports is getting stronger and stronger, which also makes the development of China's sports industry still benefit a lot.
\end{abstract}

\section{Introduction}

As the technology of image processing [1] matures, there will be more and more things we can do with this technology. We can also apply this technology to various sports [2]. In the training guidance, use this technology to check the characteristics of specific sports training [3] to check whether the action meets the standard to make prompts to achieve the purpose of improving the quality of various sports training. We can use this technology to identify the image information collected during sports [4], identify the training actions in the sports, and then combine the feature analysis methods in the visual space of the relevant space to analyze the various sports characteristics in the sports. Through image feature analysis and edge feature detection technology [5], the main features of sports training sports images are extracted. Edge contour detection [6] and corner detection technology realize motion capture in sports training [7], aiming at actual training in various sports [8]. Research on the application of capture methods in sports training has received widespread attention. The capture action of a specific motion is based on the collection and characteristic analysis, the dynamic characteristics of the training actions are extracted, and then the three-dimensional image is reconstructed [9], using the motion training motion capture combined with the spatial region [10] reconstruction method to improve the training actions of each sport capture ability. A motion recognition method based on image processing [11] is proposed here. First, collect sports training action images, capture sports training actions in Gaussian blur [12] affine space [13], and extract features. Use image processing methods to realize the recognition and optimization of sports training actions [14]. Finally, a simulation test [15] and analysis were carried out, and the validity conclusion was reached. For the development of the entire sports industry in today's society [16], the existence of this technology is also very necessary. This technology can better assist athletes in training. Through the study of the existing literature, it is found that, in the mature stage of image processing technology, the recognition and training of sports 
behavior can improve the effect of sports. However, the accuracy and effect of recognition are not ideal, and the guidance of reasonable training behavior is limited. Using image processing technology to recognize sports behavior is the main way to improve the effect of sports in the future. Therefore, image processing technology is also of great significance to the layout of sports industry structure.

\section{Image Processing Technology Concepts and Methods}

Image processing technology uses various devices that can take pictures or produce pictures to generate images and then use this technology to identify or process these images. This technology mainly includes image digitization, image enhancement and restoration, image data encoding, image segmentation, and image recognition.

The image processing technology specifically includes point processing, group processing, geometric processing, and frame processing. Since the processing target is a pixel, the most basic method among these methods is the point processing method. This method of treatment is simple and effective. Its main function is to adjust the brightness of the image, adjust the contrast of the image, invert the brightness of the image, and so on. Because the processing method of the image group has a wider processing range than the method of point processing, it is also called "area processing or block processing." For group processing, the main application of this method in image recognition covers the detection and enhancement of the edge of the relevant image, the softening of the uploaded image, and the sharpening of the uploaded image, increasing or reducing the noise of the uploaded image, and so on. The geometric processing method of the uploaded image is to use operations to change the position and arrangement order of the pixels of the uploaded image to achieve a series of effects such as adjusting the image, rotating the image, mirroring the image, changing the image. The image frame processing method synthesizes an image or multiple images to generate a new image in a specific format. Among them, the specific forms include the image generation of "number multiplication operation," the generation of "logical OR" operation relations, the generation of "exclusive OR" logical operation relations, the image generation of addition and subtraction operations, and the generation of related conditions. Image processing software usually has an image composition function, which can generate images into a variety of specific formulas.

\subsection{Classification of Image Processing Technology. Image} processing technology is generally divided into two categories: analog image processing and digital image processing.

Analog image processing includes optical and electronic processing such as photography, remote sensing image processing, and television signal processing. The characteristics of analog image processing are parallel processing and high-speed processing, which can usually reach the theoretical speed of light. Real-time TV screens are the main example of processing analog signals that can process video at a speed of 25 frames per second. Analog image processing has the shortcomings of low precision and low flexibility, and this shortcoming makes it more difficult to realize the judgment and processing ability related to nonlinearity. This technology can also be called computer image processing because it usually uses real-time computer processing or hardware processing. It also has the advantages of higher precision during processing, relatively richer processing content, better nonlinear processing, and better flexibility. Generally speaking, as long as you change the software, you can change its role. The disadvantage is that speed is still an issue, especially for complex tasks. Generally, when processing most still images, real-time processing of generalprecision digital images will require about 100 MIPS of processing power, and this will also lead to a certain degree of image resolution and accuracy. Limits: for example, the accuracy of the usual image is $1024 \times 1024 \times 12$-bit size. For this higher accuracy and resolution, the time required for processing the image is increased to a certain extent as shown in Figure 1.

Analog image processing uses lenses to achieve processing, such as photography, remote sensing image processing, and television signal processing. Analog image processing is characterized by high speed and generally realtime processing; theoretically speaking, it can reach the speed of light and can be processed in parallel at the same time. Television pictures are a typical example of analog signal processing, which deals with moving pictures at 25 frames per second. The disadvantage of analog image processing is poor accuracy and flexibility, and it is difficult to have the ability of judgment and nonlinear processing.

Digital image processing is generally done by computer or real-time hardware. Its advantages are high processing precision, rich processing content, complex nonlinear processing, and flexible flexibility. Generally speaking, the processing content can be changed by changing the software. Its disadvantage is that processing speed is still a problem, especially for complex processing. Still, pictures are mostly processed. If digital images with general accuracy are processed in real time, it is necessary to have a processing capacity of 100 MIPS. Secondly, the resolution and precision are still limited. For example, the general-precision image is $512 \times 512 \times 8$ bits, and the high-resolution image can reach $2048 \times 2048 \times 12$ bits. If the precision and resolution are higher, the processing time will be significantly increased.

\section{Sports Action Recognition Based on Image Processing Technology}

When performing this program of sports recognition based on image processing technology, you first need to collect the athlete's image, extract the motion characteristics, and then use the formula to recognize the action. When the recognition is completed, we choose a specific action to compare with the standard action, judge whether the action is standard or not, and then give specific action prompts. The athletes then modify and optimize the actions according to 


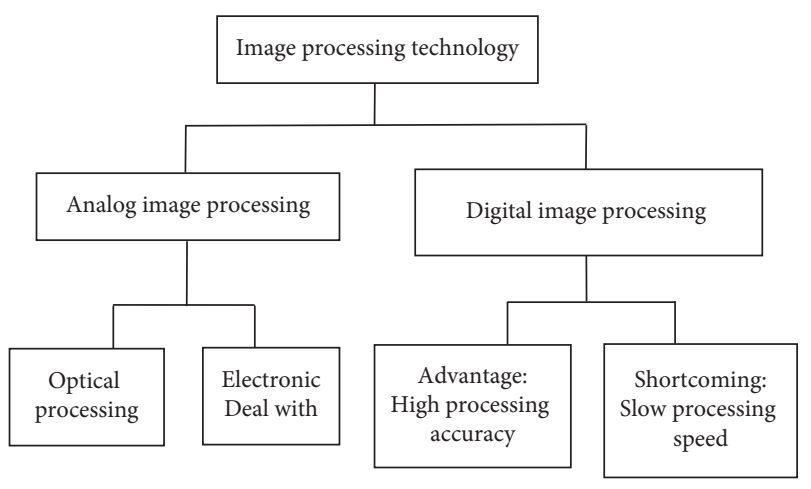

FIgURE 1: Classification of image processing technologies.

the exercise prompts to achieve better and more efficient sports results, as shown in Figure 2.

3.1. Image Acquisition. In order to obtain sports action images and perform edge contour detection on the collected high-resolution sports action images, it is first necessary to use multiresolution image scanning technology to collect training action images based on the characteristics of the $3 \mathrm{D}$ model. Assume that the function of the image of the sport is $f(x, y)$, and the background component function of the sports image is $g(x, y)$. We can use the corresponding points of 2D sports images or 3D sports images to perform model matching on $g(x, y)$ and then add Gaussian noise and Gaussian blur features to divide the image model into a 3-by-3 topology structure. Get the result shown in Figure 3.

The $3 \mathrm{D}$ image is recreated according to the distribution of Figure 3 and the $3 \mathrm{D}$ points corresponding to the $3 \mathrm{D}$ model. The distribution of the main features in the image acquisition is $n(x, y)$, and the pixels $f(x, y)$ around the current feature point are

$f^{\prime}(x, y)= \begin{cases}g(x, y)-1, & \text { if } g(x, y)-f_{\text {Lee }}^{\prime}(x, y) \geq t \\ g(x, y)+1, & \text { if } g(x, y)-f_{\text {Lee }}^{\prime}(x, y)<t \\ g(x, y), & \text { else. }\end{cases}$

Pattern matching is performed on the center pixel of the multiresolution video. Image feature decomposition modeling combined with edge contour features is described by $H$ and $n$. With the quantitative information of $H$ and $n$, the stronger the ability to search for sports actions, the closer $f$ ' $(x, y)$ will be to $f(x, y)$. On this basis, the structure model obtained by hierarchical registration is as follows:

$$
g(x, y)=h(x, y) * f(x, y)+n(x, y) .
$$

In the above formula, $H(x, y) * f(x, y)$ is the main feature point of sports training action positioning, and the symbol * represents convolution. Locate the motion image features in different regions, and obtain the affine invariant moments as

$$
g(x, y)=f(x, y)+n(x, y) .
$$

In the above formula, $n(x, y)$ is the noise interference term. Realize the capture and acquisition of sports training movements through fine registration.

3.2. Feature Detection and Extraction. We can perform relevant contour detection on the collected high-resolution sports training images, using the $3 \mathrm{D}$ model reconstruction area segmentation method of the sports training sports and the residual component mixing method, as well as the boundary feature points of the sports training and the image of the sport. Then, the vector quantization decomposition of the sports training action image is carried out, and the grayscale matching value of the sports training image is

$$
\begin{gathered}
L_{0}(r)=\frac{L(r / 2)}{2}, \\
H_{0}(r)=H\left(\frac{r}{2}\right) .
\end{gathered}
$$

In formula (4), $r$ and 0 are the decomposition coefficients of hierarchical features. When the corresponding variable satisfies $n_{m}(x, y) \in\{-1,0,1\}$, the gray-scale feature blending mode is used to obtain the edge component of the image edge of the sports training actions as follows:

$$
p\left(n_{m}(x, y)\right)= \begin{cases}\frac{r}{4}, & n_{m}(x, y)=-1, \\ 1-\frac{r}{2}, & n_{m}(x, y)=0 .\end{cases}
$$

In formula (5), $r$ is the corresponding value in the texture function of the sports action image, and its range is $0 \leq r \leq 1$. When the variance feature quantity of the output of the sports' image satisfies the expected normal distribution and the variance is $r / 2$, the feature quantity of the gray boundary of the image of the sport at this time is

$$
\begin{aligned}
& P_{c k}=\left(\frac{\sum_{j=1}^{c} I_{s w k}(1, j)}{c}, \frac{\sum_{j=1}^{c} I_{s w k}(2, j)}{c}, \ldots, \frac{\sum_{j=1}^{c} I_{s w k}(i, j)}{c}, \ldots, \frac{\sum_{j=1}^{c} I_{s w k}(c, j)}{c}\right), \\
& P_{r k}=\left(\frac{\sum_{i=1}^{r} I_{s w k}(i, 1)}{r}, \frac{\sum_{i=1}^{r} I_{s w k}(i, 2)}{r}, \ldots, \frac{\sum_{i=1}^{r} I_{s w k}(i, j)}{r}, \ldots, \frac{\sum_{i=1}^{r} I_{s w k}(i, r)}{r}\right) .
\end{aligned}
$$




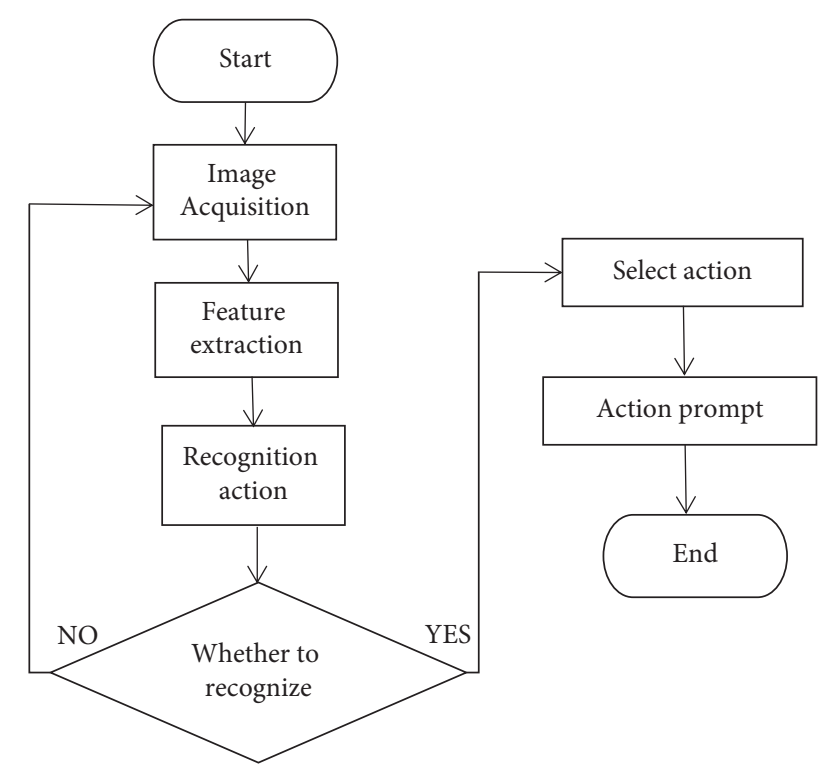

FIgURE 2: The process of sports recognition based on image processing technology.

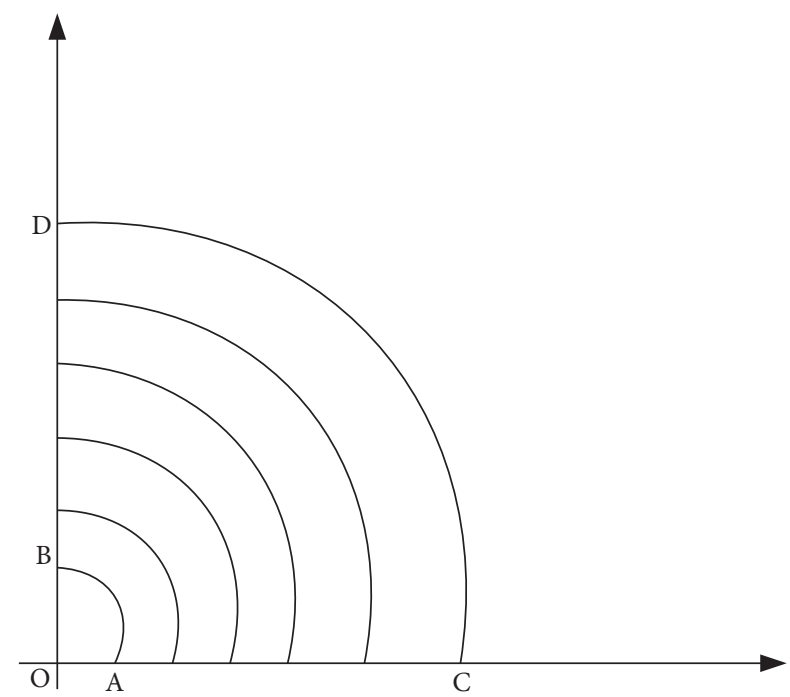

FIGURE 3: Recognition image of sports training action spatial region structure of multiframe scanning.

Based on the recognition of sports images and the contours of the edges of sports training actions, the corresponding refinement and optimization recognition are carried out. Combined with the irregular triangulation model, the corresponding theory of the visual obstruction model can be realized for sports training actions. The known generation data must be a nondecreasing and increase sequence, and the development trend of the gray quantity accumulation process can be seen through accumulation so that the law contained in the original data is displayed, and the law in the data can be summarized and utilized. The gray feature mixture model is to input a certain motion action of the motion standard as the contrast between the edge and the boundary.
The regional feature distribution model of irregular triangulation is

$$
\begin{aligned}
I_{\mathrm{GSM}} & =I\left(C^{N}, \frac{D^{N}}{s^{N}}\right) \\
& =\sum_{i=1}^{N} I\left(C_{i} ; \frac{D_{i}}{s_{i}}\right) \\
& =\sum_{i=1}^{N}\left(h\left(\frac{s_{i}}{D_{i}}\right)-h\left(\frac{D_{i}}{C_{i} s_{i}}\right)\right) .
\end{aligned}
$$

Since the correction scale of each image is different, the recognized internal texture and edge features of these images will be rearranged linearly. The image improvement results are as follows:

$$
p(x, t)=\lim _{\Delta x \longrightarrow 0}\left[\delta \frac{u-(u+\Delta u)}{\Delta x}\right]=-\delta \frac{\partial u(x, t)}{\partial x} .
$$

$\sigma$ can be expressed as the texture distribution of the image. $\Delta x$ represents the pixel value of the image gradient and predicts the local boundary and local area of the sports training image. $t(x)$ represents the image of the statistical distribution value of the sports training pixel. The specific feature detection result is expressed as

$$
L\left(a, b_{m}\right)=\sum_{V_{m} \in P^{\text {res }}} \sum_{V_{n} \in P_{\text {true }}} \frac{V_{m} \cap V_{n}}{V} \log \left(\frac{V V_{m} \cap V_{n}}{V_{m} V_{n}}\right) .
$$

In the nonlinear change mode of sports, solve the characteristic trajectory equation of sports:

$$
L\left(a, b_{m}\right)=\sum_{V_{m} \in P_{\text {res }}} \frac{V_{m}}{V} \log \left(\frac{V_{m}}{V}\right)+\sum_{V_{m} \in P^{\text {true }}} \frac{V_{n}}{V} \log \left(\frac{V_{n}}{V}\right) .
$$

Performing motion capture and feature recognition of sports in Gaussian blur, the optimized trajectory equation can be obtained as

$$
L\left(a, b_{m}\right)=\frac{1}{\sqrt{a}}\left(\frac{a+1}{2}-\frac{(a-1) f_{0}}{b}\right)
$$

Among them, $(x, y)$ represents the distribution of a group of blurred pixels in the image, and the motion capture is performed through the spatial matching of pixel blocks.

\subsection{On the Realization of the Spatial Visual Feature Analysis} Algorithm. Relevant image enhancement technology is used to improve the resolution and adaptability of the training action capture in sports, and the computer vision image processing method is used to realize the $f\left(g_{i}\right)$ texture distribution function of the athlete:

$$
f\left(g_{i}\right)=c_{1} \lambda_{i} \frac{\sum_{j=0}^{N_{n p}} \rho_{j} v_{i j} / v_{i j}^{\sigma 1}+\varepsilon}{\sum_{j=0}^{N_{n p}} \rho_{j} / v_{i j}^{\sigma 1}+\varepsilon} .
$$


From the above, the dynamic distribution of the trajectory in the sports training actions can be obtained, and the specific expression is

$$
f\left(G_{n}\right)=a_{1}+a_{2} x+a_{3} y+a_{4} z+\sum_{i=0}^{n} \gamma_{i} U\left(g_{i}, p_{i}\right),
$$

where $\phi\left(T_{n}\right)$ is obtained by the following formula:

$$
\phi\left(T_{n}\right)=\gamma^{t} H \gamma+\theta^{T} H \theta+\omega^{T} H \omega .
$$

Through the above steps combined with the method of reconstructing the trajectory of its sports movement, the expression is as follows:

$$
G_{\text {new }}=(1+\mu T)(1+\lambda T) G_{\text {old }} .
$$

In equation (15), $G_{\text {new }}$ and $G_{\text {old }}$, respectively, represent the gray track components of sports training actions:

$$
\text { Is_visible }\left(\mathrm{M}_{\mathrm{mi}}, \mathrm{C}_{\mathrm{j}}\right)= \begin{cases}1, & \text { if }\left\{\begin{array}{l}
j \neq i, \\
\mathrm{C}_{\mathrm{j}} \text { maycallM }_{\mathrm{mi}},
\end{array}\right. \\
0, & \text { otherwise. }\end{cases}
$$

In formula (16), $c$ is the decomposition formula of the size feature of the image of the sports action and $\operatorname{Md}(C i)$ is the specific information component in $\mathrm{Ci}$. According to the above description, image recognition technology can be used to achieve the optimization of capturing characteristic actions during sports training.

According to the aforementioned spatial visual feature analysis algorithm, standard actions can be compared with sports actions in the collected picture information, and areas that are not standard enough can be proposed and then optimized based on this to achieve better training results.

3.4. Experimental Comparison. According to the information in the above article, we can compare the accuracy rate achieved by this algorithm with other algorithms. Of course, it must be on the premise of the same image quality. If the image quality is different, it will be compared. This comparison will be meaningless. If the quality of the image we collect is high, the accuracy and correctness of the sports feature actions we can recognize in the image will be higher. Different types of pictures are compared using the same algorithm.

There are some differences between different algorithms, which will cause the accuracy of the results of the final algorithm to be different. We can use the feature capture algorithm to achieve sports action recognition and do not use the algorithm or use the comparison chart of the accuracy of sports action recognition performed by other algorithms as shown in Figure 4.

In the entire spatial visual feature analysis algorithm, several indicators that can be used as evaluation indicators for this algorithm: accuracy, response speed, feasibility, clarity, and ambiguity. Based on these evaluation indicators, when we want to use this technology to complete a certain related work, we can use these indicators for comparison to select the more important indicators for ourselves for
Accuracy of identifying features when image quality is different

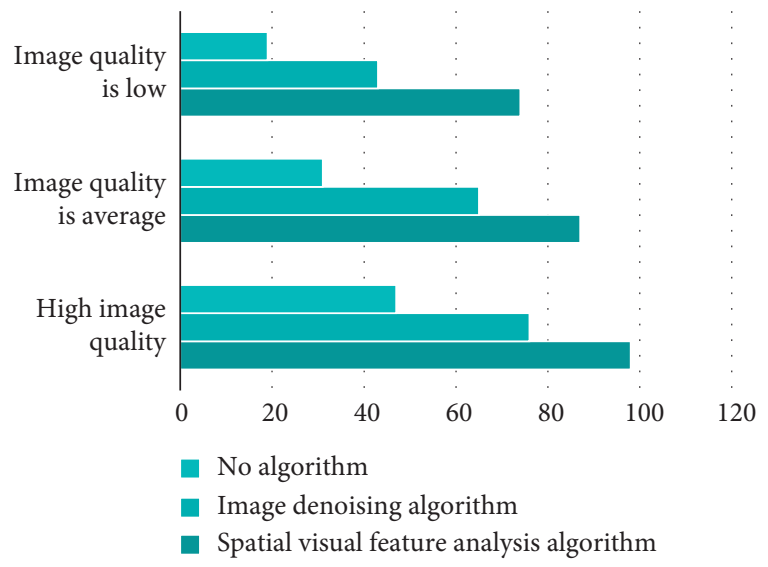

Figure 4: The accuracy of identifying features when the image quality is different.

evaluation and finally choose the algorithm, fundamentally, saving working time in Figure 5.

Comparing the recognition rate of the spatial vision feature algorithm in this article with the mainstream algorithms at home and abroad on the MSRGcsturc3D database, it can be seen that the spatial vision feature analysis algorithm in this article uses the texture distribution function and a series of other functions, which greatly improves the recognition rate of image acquisition, and the improvement of recognition rate also improves the efficiency of image recognition to a certain extent in order to achieve the purpose of reducing the running time of the algorithm as shown in Table 1.

When collecting images, the body parts covered in the images are different, and the experimental results we can get will be slightly different. For example, when recognizing legs and arms, the key feature points of the image we are targeting are different. Differently, in this case, we should first understand the key features of the corresponding arm or leg and then perform specific feature positioning based on these features to improve the accuracy of identifying a specific part rate and achieve the purpose of saving time.

When implementing a specific sports action recognition algorithm based on image processing technology, the specific information in the image may be different, and the feature recognition of the specific action may also be different. For the same image, it may be that the legs in the image are recognized, and it is also possible to recognize the arms or faces in the image and other parts. For these different parts to be identified, the amount of feature that needs to be extracted may also be different as shown in Figure 6 .

In the entire sports action recognition algorithm based on image processing technology, the number of times each body part is extracted is also very different. Moreover, the number of times of extraction will be different because of the different objects to be extracted. For athletes, every part of the body will be extracted for comparison. For ordinary people, in most cases, only the legs or arms need to be extracted and then compared, as shown in Figures 7 and 8. 


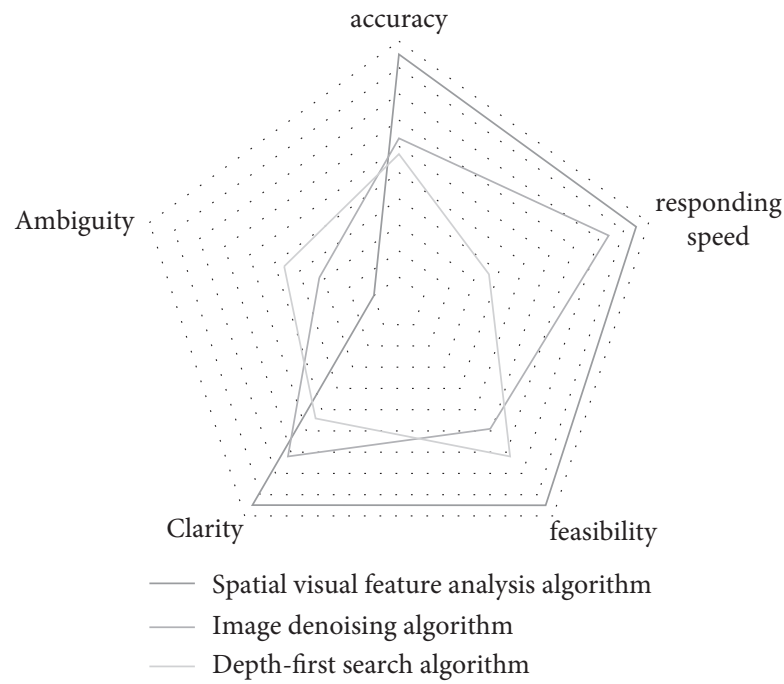

FIGURE 5: Comparison of evaluation indicators among various algorithms.

TABLE 1: Comparison of the recognition rate of the algorithm in this paper with the mainstream algorithms at home and abroad on the MSRGcsturc3D database.

\begin{tabular}{lc}
\hline Method & Recognition rate (\%) \\
\hline Action graph on occupancy & 80.50 \\
Action graph on silhouette & 87.70 \\
Random occupancy pattern & 88.50 \\
Depth motion maps & 89.20 \\
HON4D & 92.45 \\
Spatial visual feature analysis algorithm (method in this article) & 94.17 \\
\hline
\end{tabular}

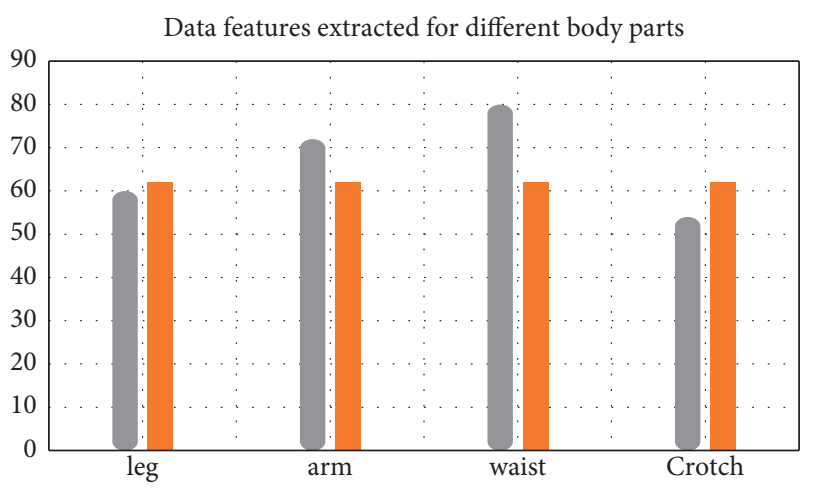

The amount of features to be extracted Average extraction

FIGURE 6: Data features extracted for different body parts.

\section{Development and Analysis of Sports Industry Pattern}

Nowadays, the sports industry is the carrier of China's economic development. It has the characteristics that other industries also have; that is, it pays attention to the benefits of the market and the economic benefits of today. Differentiating characteristics: the products of this industry have the function of improving the physical fitness of residents, promoting the spirit of famous people, and realizing the overall development of individuals and the progress of social civilization.

According to the new statistical classification of sports industry, China's sports industry can be divided into sports product and related product manufacturing, sports service industry, and stadium construction industry. Among them, the sports service industry includes several subcategories such as sports management activities, sports competitions and performance activities, sports fitness and entertainment activities, and sports facilities and facility management as shown in Figure 9.

For the development of the sports industry, the Chinese government is also giving full support, and all relevant units have also issued favorable documents in terms of funds and policies. This makes the growth of the total scale of China's sports industry steadily increasing as shown in Figure 10.

In addition, the structure of China's sports industry is gradually optimized, and China's sports industry is dominated by the manufacturing of sports equipment and related products. In recent years, China has strongly supported the development of the sports industry. In 2018, the proportion of scale reached $47.9 \%$, the proportion of sports equipment and related industries in the entire sports industry gradually declined, reaching $49.7 \%$ in 2018 , and the gap has been increasing year by year. 


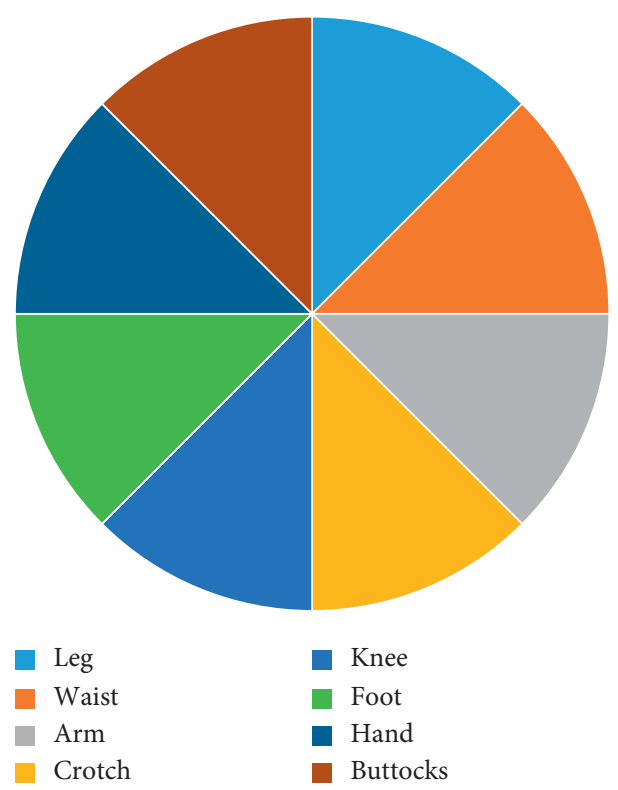

Figure 7: The distribution of the number of feature extraction required by each body part for athletes.

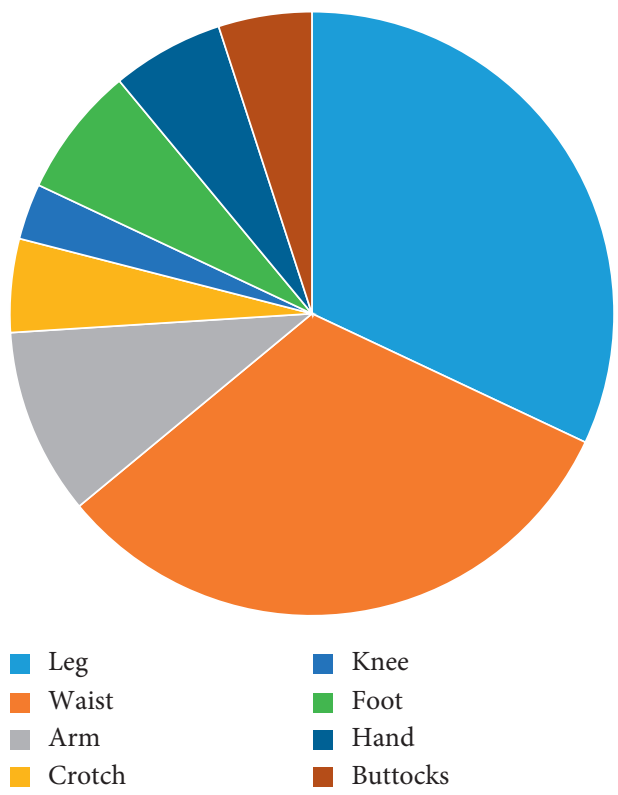

FIGURE 8: The distribution of the number of feature extractions required by each body part for ordinary people.

It can also be seen from the industrial added value that since 2016, the proportion of the added value of China's sports service industry has surpassed that of the sports goods manufacturing industry and related industries. In 2018, the added value of the sports service industry was approximately twice that of sports goods and related industrial production as shown in Figures 11 and 12.

In the entire Chinese sports industry, the proportion of various industries in the entire sports industry is different. As far as today is concerned, the sports service industry accounts for the largest proportion of the entire sports industry, followed by sports, the manufacturing of supplies and related products, then the others, and finally the construction of sports venues, as shown in Figure 13.

Based on the rapid development of the entire sports market, and because of the commercialization of today's events, the scale of the relevant sports event market is also growing rapidly. However, due to the lack of traffic, this growth rate is not very fast. In general, the entire market still has long-term investment value, as shown in Figure 14.

Consumption in the sports market in the current era has also led to the rapid growth of per capita sports 


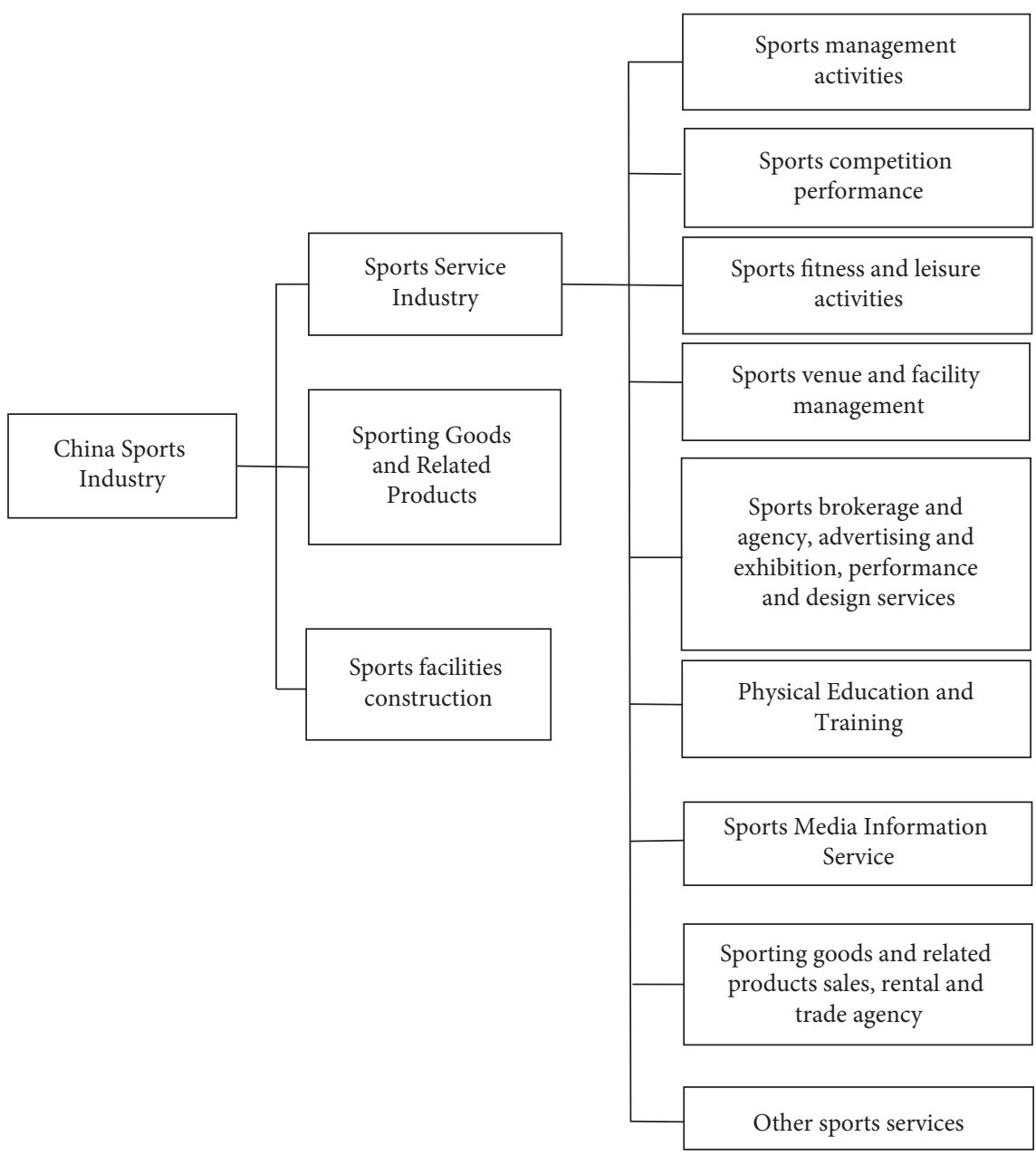

FIGURE 9: Classification of China's sports industry.

The total scale of China's sports industry from 2015 to 2019 (unit: 100 million yuan)

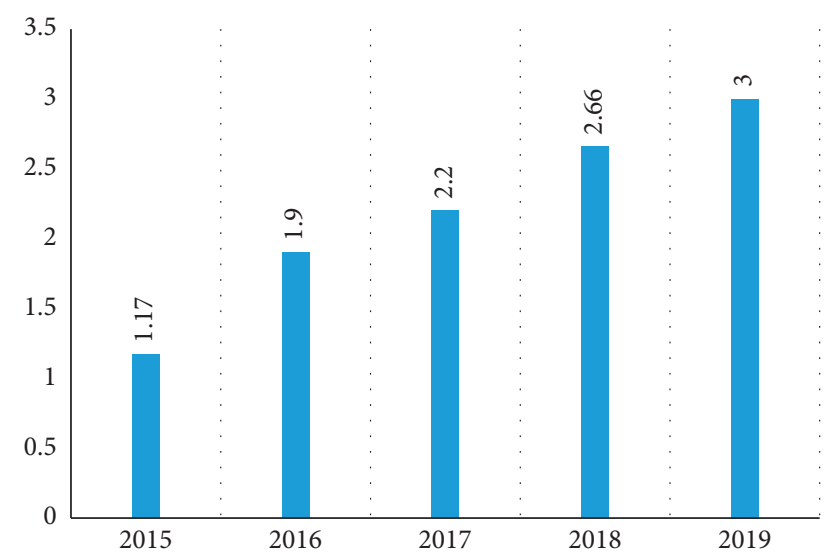

FIgURE 10: The total scale of China's sports industry from 2015 to 2019 (unit: 100 million yuan).
The proportion of the total scale of China's sports industry segmentation industry from 2015 to 2019 (unit: \%)

120

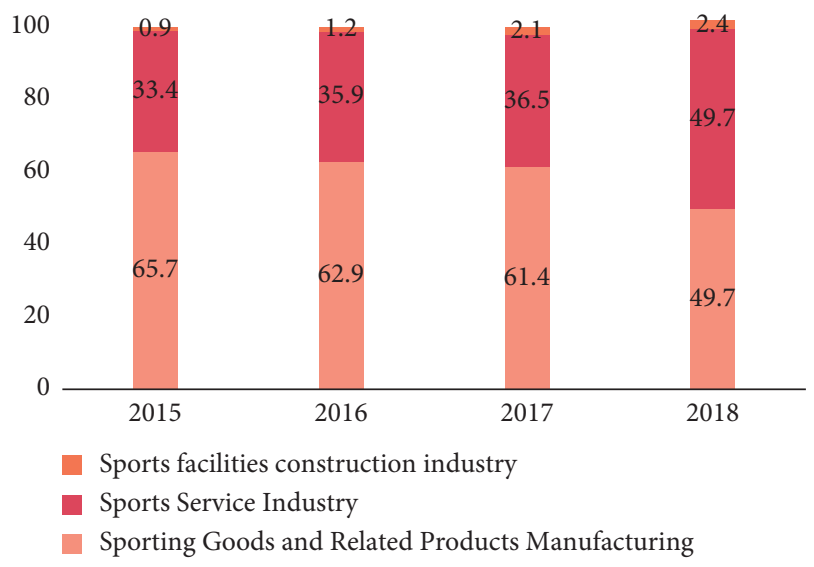

Figure 11: The proportion of the total scale of China's sports industry segmentation industry from 2015 to 2019 (unit: \%). 
The proportion of the added value of China's sports industry segmentation industry from 2015 to 2019 (unit: \%)

120

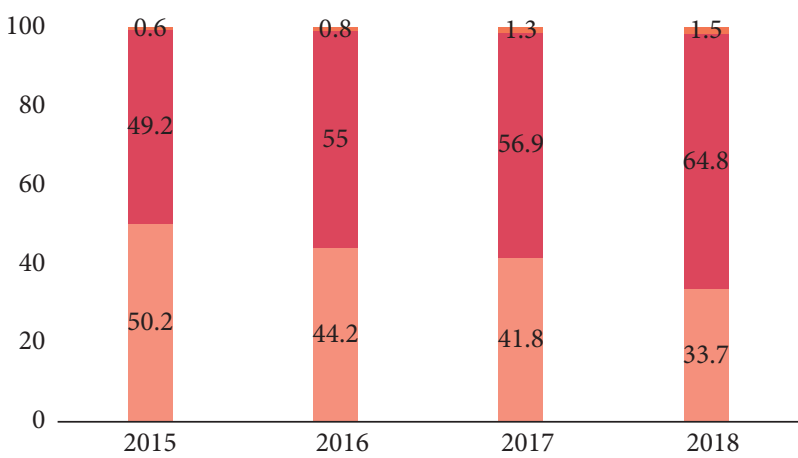

Sports facilities construction industry

Sports Service Industry

Sporting Goods and Related Products Manufacturing

Figure 12: The proportion of the added value of China's sports industry from 2015 to 2019 (unit: \%).

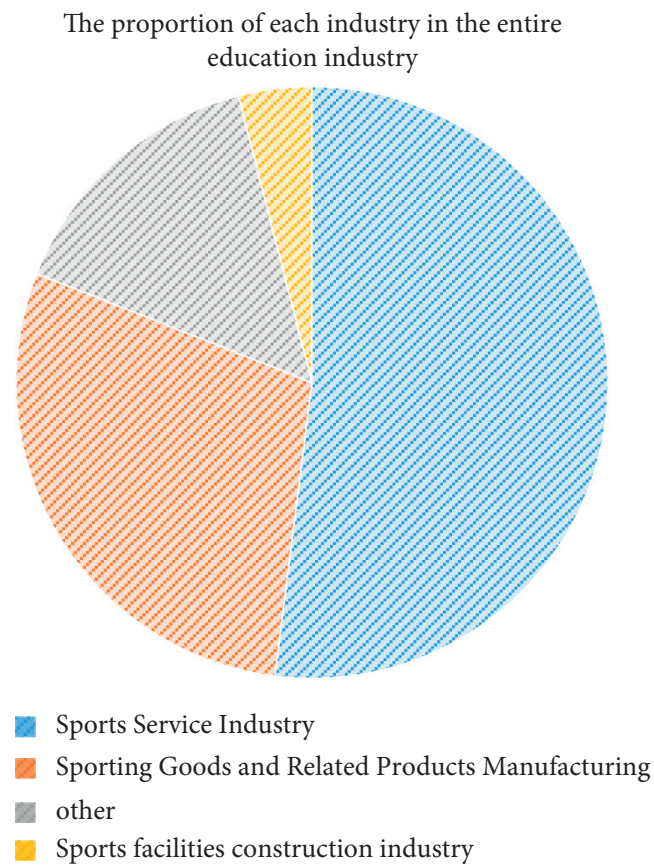

FIGURE 13: The proportion of each industry in the entire education industry.

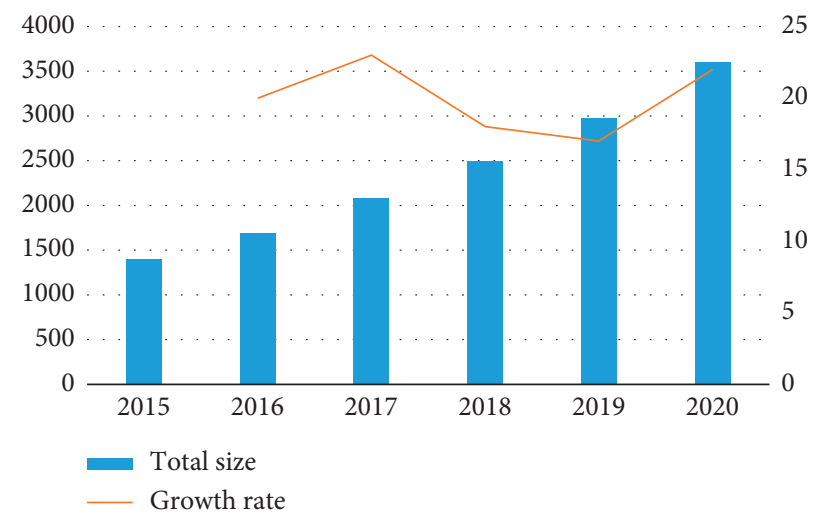

FIGURE 14: Market scale and growth rate of domestic sports events. 


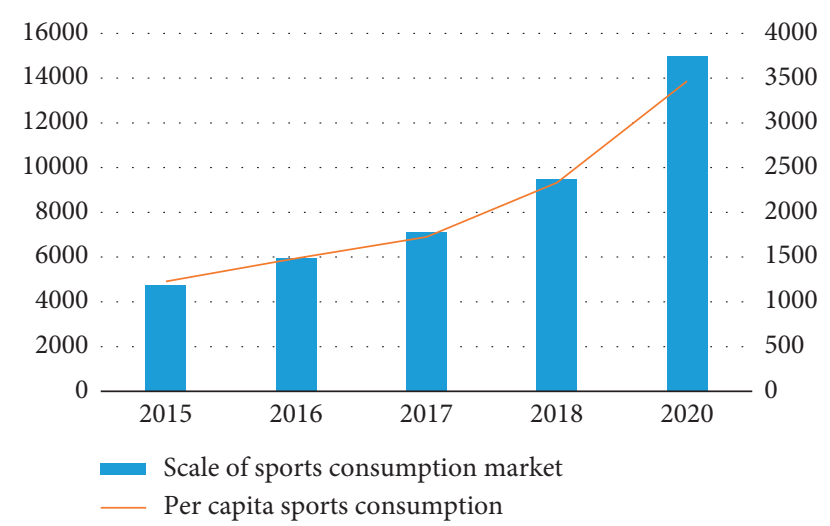

FIgURE 15: China's sports consumption market and per capita sports consumption growth.

consumption. In 2018, the scale of China's sports market had grown to nearly one trillion yuan. By 2020 , driven by the rapid growth of per capita sports consumption expenditure, China's sports consumption market is expected to reach 1.5 trillion yuan. The mainstream sports market in China has grown from 593 yuan in 2011 to 2,264 yuan. It is expected to reach 3448 yuan in 2018, with an annual compound interest rate of $19.24 \%$ in the past ten years, as shown in Figure 15.

\section{Conclusion}

In today's era, the rapid development of information makes our forms of life or work become more diversified. Take the aspect of sports in our article. If the technology of sports action recognition based on image processing technology can be used properly, this technology can help athletes assist in training. Of course, this technology can also be used in our ordinary people's lives. For us, we only need to apply this technology to fitness. It can be used to check whether our exercises are standard during fitness to achieve better training results. It can also prevent us from hurting ourselves during exercise. Furthermore, the development of the current sports industry is also becoming more and more mature, which will make the existence of this technology more necessary and meaningful. According to relevant information, China's future urban sports industry must take the road of high-quality development. The urban development in the development of the sports industry must focus on the combination of software and hardware. At the same time as the hardware construction, it must also focus on improving the quality of services and pay more attention to the needs of local residents. To develop the sports industry, we must clearly recognize the internal and external relationships. Looking inwardly, the sports industry is essentially a livelihood undertaking and a happiness project. In the final analysis, it must serve the local people. At the same time, the development of the sports industry is not an overnight event but a long-term process. The key is to cultivate healthy living habits and living habits. Today, the future of China lies in young people, so for us, training the exercise habits of young people is the most important thing. Let young people fall in love with sports and eventually move towards a healthy life of sports for all to fundamentally meet the needs of the development of the sports industry.

\section{Data Availability}

The experimental data used to support the findings of this study are available from the corresponding author upon request.

\section{Conflicts of Interest}

The authors declare that they have no conflicts of interest regarding this work.

\section{References}

[1] H. Kim, W.-K. Jung, Y.-C. Park, J.-W. Lee, and S.-H. Ahn, "Broken stitch detection method for sewing operation using CNN feature map and image-processing techniques," Expert Systems with Applications, vol. 188, Article ID 116014, 2022.

[2] F.-G. Juan Pedro, C.-M. Daniel, and E.-L. Fernando, "Physiological benefits of digital applications in health and sport performance," Physiology \& Behavior, vol. 242, Article ID 113619, 2021.

[3] E. M. Crowe, J. B. J. Smeets, and E. Brenner, "The response to background motion: characteristics of a movement stabilization mechanism," Journal of Vision, vol. 21, no. 11, p. 3, 2021.

[4] L. Lu, Y. Wang, X. Cai, and X. Hu, "A binocular reconstruction method fused with Laplacian image information for pavement texture evaluation," Measurement, vol. 185, Article ID 107638, 2021.

[5] P. Bagavac, L. Krstulović-Opara, and Ž. Domazet, "Enhancing IR thermographic inspection of subsurface defects by using the technique of edge detection," Russian Journal of Nondestructive Testing, vol. 57, no. 7, pp. 609-618, 2021.

[6] D. Li, L. Bei, J. Bao, S. Yuan, and K. Huang, "Image contour detection based on improved level set in complex environment," Wireless Networks, vol. 27, no. 7, pp. 4389-4402, 2021.

[7] N. Jia and C. Zheng, "Two-level discriminative speech emotion recognition model with wave field dynamics: a personalized speech emotion recognition method," Computer Communications, vol. 180, pp. 161-170, 2021.

[8] R. C. Koch, D. Faurholt-Jepsen, C. Ritz et al., "The impact of physical training on length of hospital stay and physical function in patients hospitalized with community-acquired pneumonia: protocol for a randomized controlled trial," Trials, vol. 22, no. 1, p. 571, 2021.

[9] J. Sun, "A 3D image encryption algorithm based on chaos and random cross diffusion," Modern Physics Letters B, vol. 35, no. 30, 2021.

[10] M. Q. Rieck and B. Wang, "Locating perspective three-point problem solutions in spatial regions," Journal of Mathematical Imaging and Vision, vol. 63, no. 8, pp. 953-973, 2021.

[11] K. B. de Carvalho, V. T. Basílio, and A. S. Brandão, "Action recognition for educational proposals applying concepts of Social Assistive Robotics," Cognitive Systems Research, vol. 71, pp. 1-8, 2022. 
[12] L. Fu, J. Zhu, W. Li et al., "Tunnel vision optimization method for VR flood scenes based on Gaussian blur," International Journal of Digital Earth, vol. 14, no. 7, pp. 821-835, 2021.

[13] W. Paweł, "Parallel locally strictly convex surfaces in fourdimensional affine space contained in hyperquadrics," Symmetry, vol. 13, no. 9, p. 1575, 2021.

[14] N. Sharma and U. Batra, "An enhanced Huffman-PSO based image optimization algorithm for image steganography," Genetic Programming and Evolvable Machines, vol. 22, pp. 189-205, 2021.

[15] X. Yang, "Exploration and practice of online and offline mixed teaching in functional experiment teaching," International Journal of Social Science and Education Research, vol. 4, no. 10, 2021.

[16] D. G. Das, "Research on sports industry governance mode," Journal of Social Science and Humanities, vol. 3, no. 8, 2021. 